\title{
Multicentric Hemangiopericytoma of Posterior Fossa: A Rare Case and Review of Literature
}

\author{
Harish Naik ${ }^{1}$ Velho Vernon ${ }^{1}$ Anuj Bhide ${ }^{1} \quad$ Amrita Guha $^{2}$ Laxmikant Bhople $^{1}$ Nimesh Jain ${ }^{1}$
}

\author{
${ }^{1}$ Department of Neurosurgery, J.J. Group of Hospitals and Grant \\ Medical College, Mumbai, Maharashtra, India \\ 2 Department of Radiology, J.J. Group of Hospitals and Grant Medical \\ College, Mumbai, Maharashtra, India
}

\author{
Address for correspondence Dr. Harish Naik, MCh, Department of \\ Neurosurgery, J.J. Group of Hospitals and Grant Medical College, \\ Mumbai, Maharashtra 400008, India (e-mail: haryadoc@gmail.com).
}

Indian J Neurosurg 2017;6:113-116.

\begin{abstract}
Keywords

- hemangiopericytoma

- surgery

- radiotherapy

Intracranial hemangiopericytoma is unusual, and those occurring in the posterior fossa are extremely rare. Surgery followed by radiotherapy is the mainstay of treatment. These tumors are known to recur locally and distant metastases are reported. Multicentricity either in the primary setting or in recurrent setting is rarely reported. We report here a rare case of multicentric posterior fossa hemangiopericytoma occurring in a 38-year-old man at two different intervals. The patient underwent total excision of right tentorial hemangiopericytoma in 2013 , followed by radiotherapy. After being free from disease for 3 years, he developed a similar lesion on left tentorial leaflet in 2016 for which a total excision was once again done followed by adjuvant radiotherapy.
\end{abstract}

\section{Introduction}

Hemangiopericytomas (HPCs) are rare aggressive tumors, thought to comprise 2 to $3 \%$ of all primary meningeal tumors and $0.4 \%$ of all intracranial tumors. ${ }^{1-5}$ It was first reported by Stout and Murray ${ }^{6}$ and occurs mostly in soft tissue, including brain meninges primarily. Histologically, intracranial HPC arises from malignant transformation of Zimmerman's pericytes, supportive cells that help form the walls of meningothelial capillaries. ${ }^{1,2,6}$ HPC is considered a World Health Organization (WHO) grade II neoplasm, with anaplastic variants classified as grade III. ${ }^{4}$ Because HPC closely mimics meningioma in clinical and radiographic presentation, histologic confirmation is the only definitive means of distinguishing the diagnosis.

Even after aggressive initial management combining gross total resection (GTR) with adjuvant external beam radiotherapy (EBRT), recurrence rates have been as high as $30 \%{ }^{3}$ These tumors have high propensity toward progression making optimal primary treatment of HPC a difficult endeavor.

received

January 16, 2017

accepted

May 2, 2017

published online

June 9, 2017
DOI https://doi.org/

10.1055/s-0037-1603682. ISSN 2277-954X.

\section{Case Report}

A 38-year-old man presented in our neurosurgery clinic in May 2013 with complaints of holocranial headache associated with vomiting and blurring of vision for a period of 8 days. On examination cerebellar signs were present. Fundoscopy showed evidence of grade III papilledema. Computed tomography (CT) of the brain demonstrated a well-defined extra-axial hyperdense intensely enhancing lesion in right cerebellopontine angle with supra- and infratentorial extension with partial filling defect in right transverse and sigmoid sinus s/o meningioma. Ventriculoperitoneal shunt was inserted. Magnetic resonance imaging (MRI) of the brain showed a well-demarcated, solid mass in the right cerebellum of approximately $4.5 \times 3.1 \times 3.3 \mathrm{~cm}$ around the transverse and sigmoid sinus area. The mass demonstrated a heterogeneous low signal on T2-weighted images and an iso-low signal on T1-weighted images with signal voids (-Fig. 1a, b). The mass was strongly enhancing. The patient underwent right retrosigmoid suboccipital craniotomy with Simpson's grade IV excision of the lesion. The

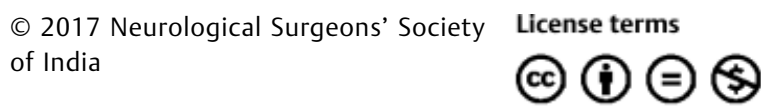



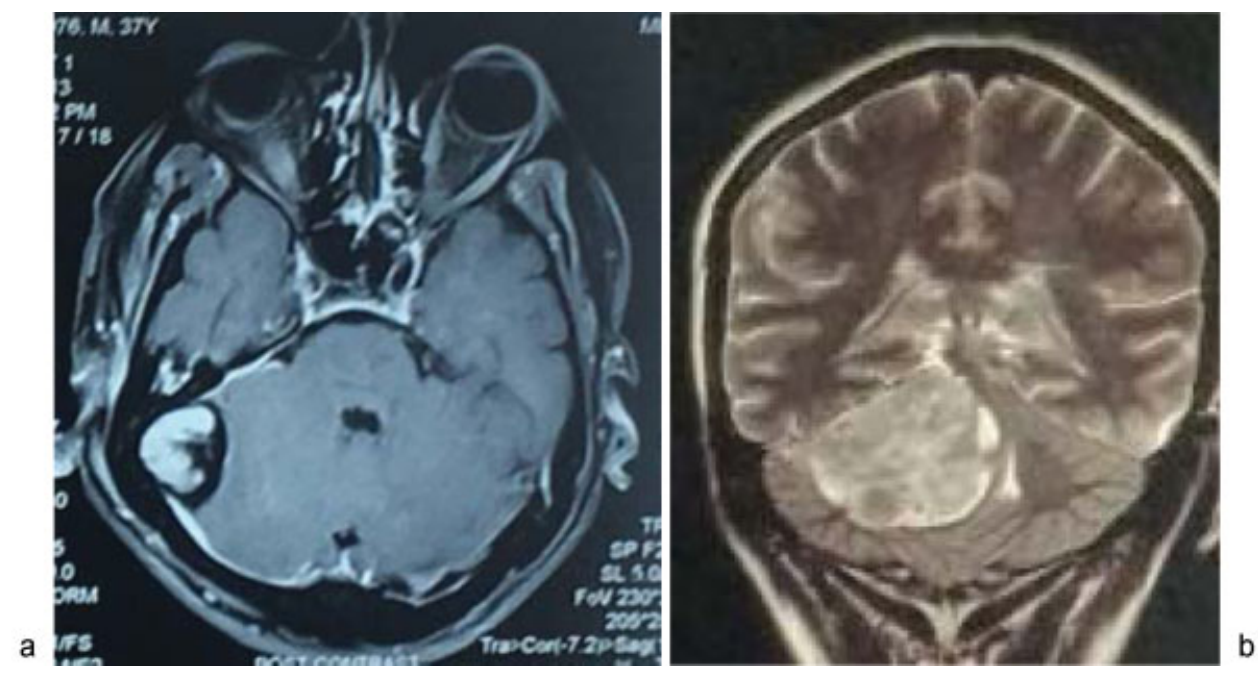

Fig. 1 (a, b) MRI showed a well-demarcated, solid mass in the right cerebellum of approximately $4.5 \times 3.1 \times 3.3 \mathrm{~cm}$ around the transverse and sigmoid sinus area. The mass demonstrated a heterogeneous low signal on T2-weighted images and an iso-low signal on T1-weighted images with signal voids suspected to be a vascular structure.

tumor was highly vascular and a part of tumor invading sigmoid sinus was left behind. Postoperative course was uneventful. Specimen examination revealed meningeal hemangiopericytoma (MHPC) WHO grade II. He received whole brain radiotherapy to a dose of $50 \mathrm{~Gy}$ in 25 fractions over 5 weeks. Follow-up MRI of the brain at 6 months showed no evidence of recurrence or residual lesion (- Fig. 2).

Three years later in 2016, the patient came back with headache and cerebellar ataxia. MRI of the brain showed a well-demarcated, solid mass in the left cerebellum of approximately $3.9 \times 3.8 \times 4.1 \mathrm{~cm}$ around the transverse and sigmoid sinus area. The mass demonstrated a heterogeneous low signal on T2-weighted images and an iso-low signal on T1-weighted images with signal voids. T1-

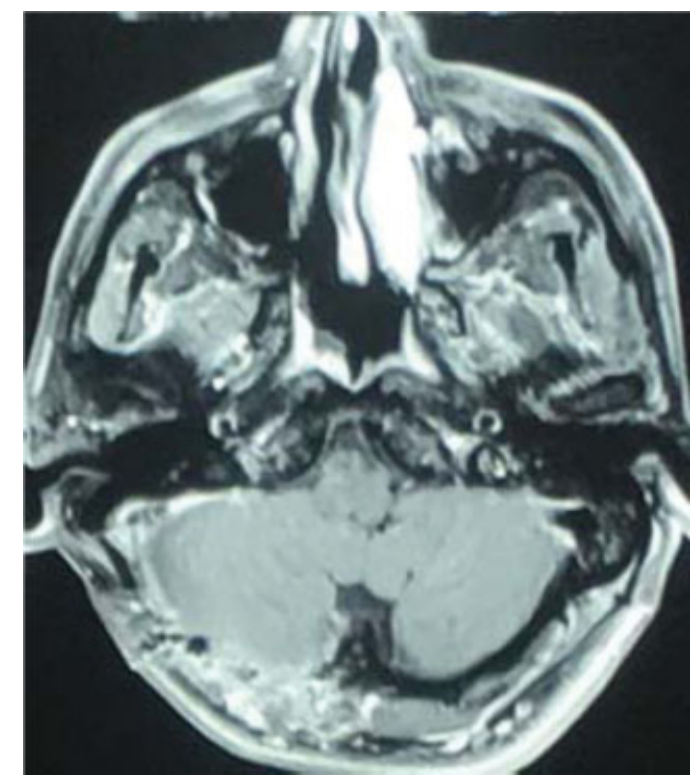

Fig. 2 Postoperative MRI of the brain at 6-month follow-up showed no residual lesion. weighted postgadolinium contrast images showed intense homogenous enhancement ( $\mathbf{- F i g . ~ 3 a , ~ b ) . ~ L e f t ~ r e t r o s i g m o i d ~}$ approach was taken. Tumor was reddish brown (-Fig. 4) highly vascular with well-defined capsule. Near-total excision was achieved. He was discharged on 10th postoperative day. Microscopically the tumor comprised numerous stag hornshaped blood vessels lined by plump endothelial cells interspersed with spindle-shaped cells having round to oval nuclei. Immunohistochemically the tumor was positive for CD34, whereas no immunoreactivity was seen for epithelial membrane antigen (EMA) or progestogen receptor. He received another course of cranial irradiation with a lower dose (40 Gy, conventional fractionation). The treatment was well tolerated. Postoperative MRI performed 2 weeks after surgery showed no evidence of residual lesion (-Fig. 5).

\section{Discussion}

Intracranial HPCs either in primary or recurrent setting are rarely reported in the literature. ${ }^{7}$ Primary intracranial HPC has mostly been found in supratentorial regions and less frequently in the infratentorium. ${ }^{7}$ Reviewing other literatures, we found a study reporting primary HPC located in the posterior fossa and cerebellopontine angle as $12 \%$ of intracranial HPCs (range: $5-21 \%$ ). ${ }^{8,9}$ Multicentric primary HPC in the posterior fossa that invades the transverse and sigmoid sinuses, such as in this case report, has rarely been reported in neurosurgical literature.

In accordance with the published literature on intracranial HPC, recurrence rates have been as high as $30 \%{ }^{1,4}$ Even in cases of apparent GTR, microscopic pockets of malignant tumor cells likely remain present, inevitably leading to recurrence. The addition of radiation to surgery in the treatment of HPC may stunt tumor growth by altering the biology of these remaining tumor cells, leaving them less likely to divide and eventually play an important 

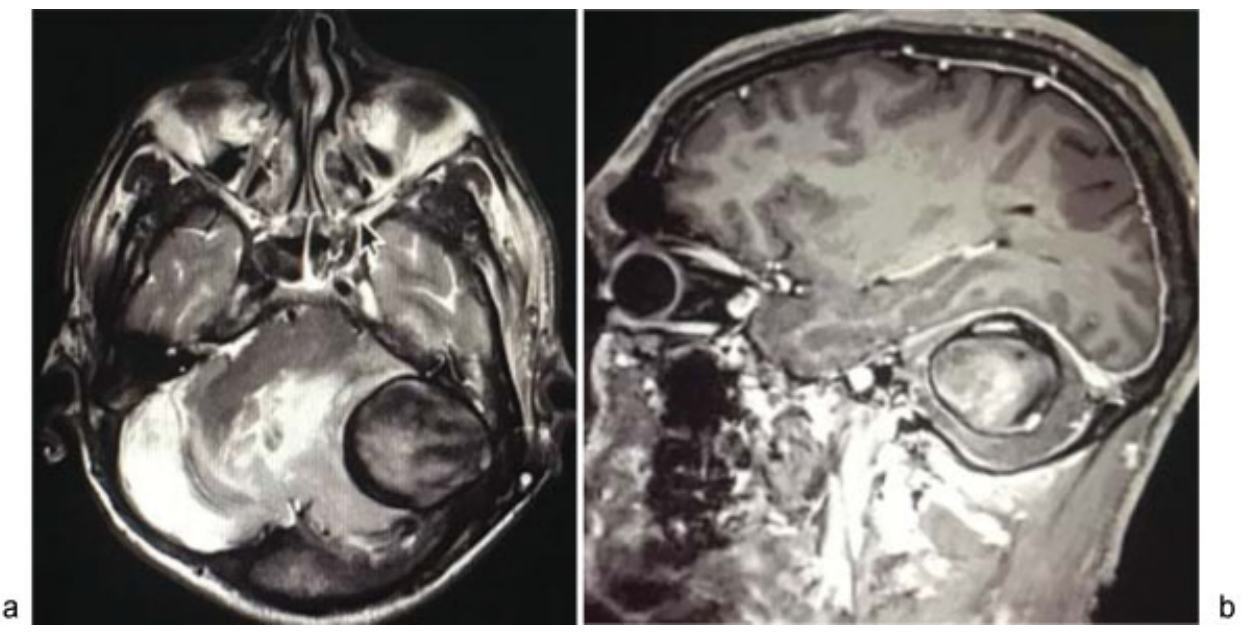

Fig. 3 (a, b) Magnetic resonance imaging (MRI) at second admission showed a well-demarcated, solid mass in the left cerebellum of approximately $3.9 \times 3.8 \times 4.1 \mathrm{~cm}$ around the transverse and sigmoid sinus area. The mass demonstrated a heterogeneous low signal on T2-weighted images and an iso-low signal on T1-weighted images with signal voids. T1-weighted postgadolinium contrast images showed intense homogenous enhancement.

role in the prevention of recurrence, and causing tumor progression.

Descriptions of the MRI features of intracranial HPC are limited. Intracranial HPCs are heterogeneous, predominantly isointense on T1- and T2- weighted magnetic resonance images, show prominent internal vessel voids, and enhance heterogeneously on contrast-enhanced magnetic resonance images. ${ }^{4}$

Macroscopically, HPCs exhibit high vascularity, with intrinsic appearance of gray color in the visible vascular spaces. Microscopically, a prominent "staghorn" vascular channel around a reticulin network is a common finding with the tumor cells round to spindle shaped. These findings differ from those of meningiomas, namely nuclear pseudo-inclusions and psammoma bodies. ${ }^{4,9}$

The most important immunohistochemical (IHC) markers thought to be diagnostically useful are CD34, Bcl-2, and CD99. CD34 is thought to be specific for HPCs. ${ }^{10} \mathrm{Bcl}-2$ and CD99 have

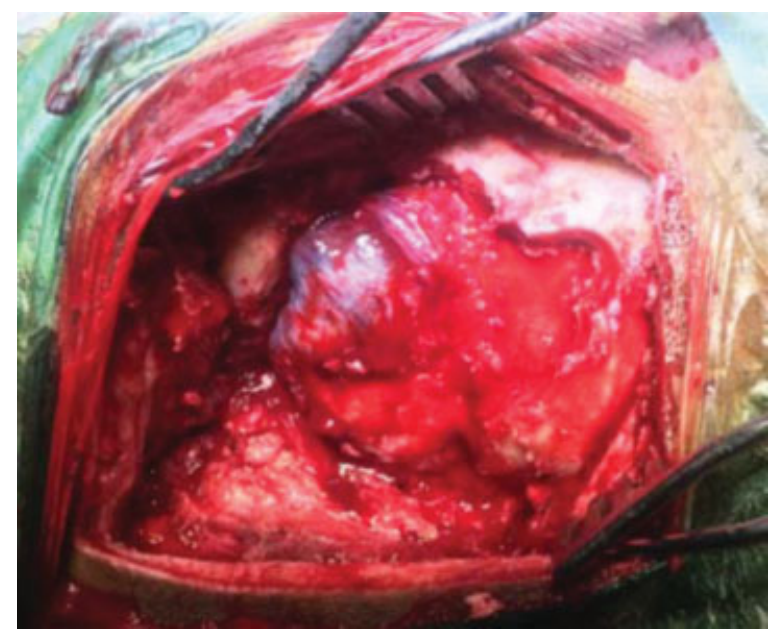

Fig. 4 Tumor was reddish brown, highly vascular with well-defined capsule and adherent to tentorium and invading transverse and sigmoid sinus. also been reported as very sensitive markers for HPCs. ${ }^{10}$ Most HPCs strongly coexpressed CD99 and Bcl-2, with this pattern being highly specific. In accordance with Shoji et al, ${ }^{11}$ most MHPCs also strongly express p21. A fusion gene between NAB2 and STAT6 has been recently identified as potential marker specific for HPCs. ${ }^{12}$ STAT6 is a highly sensitive marker for the diagnosing HPCs. Together considered as IHC STAT6, CD34, CD99, and Bcl-2 findings could provide more supportive diagnostic information.

Loss of $1 \mathrm{p}, \mathrm{NF} 2,4.1 \mathrm{~B}$, and $14 \mathrm{q}$ by fluorescence in situ hybridization (FISH) has been frequently identified in

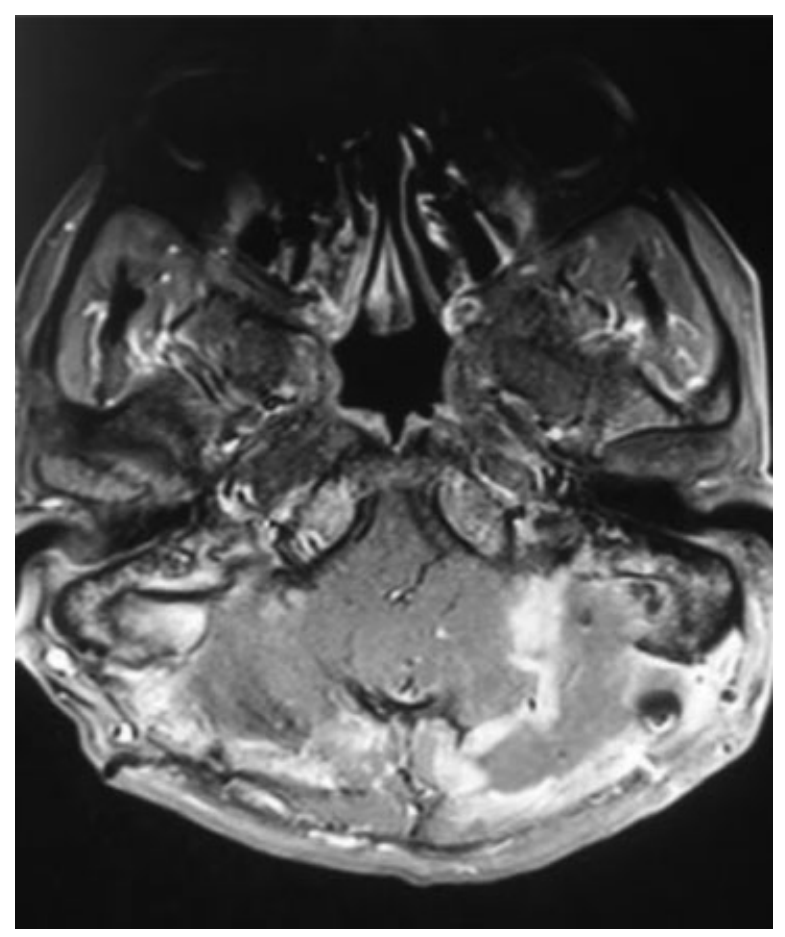

Fig. 5 Postoperative MRI after second surgery showed no residual lesion. 
meningiomas, whereas no consistent chromosomal losses or gains have been seen in HPCs.

Considering the aggressive nature of HPCs, GTR of the tumor is the optimal treating method. ${ }^{3,4}$ It has been demonstrated that local treatment is strongly related with the final prognosis, but the prognosis remains dismal due to the unpredictable malignancy of HPC. ${ }^{13}$

Though surgery is the definitive modality of treatment, radiotherapy has been used to treat surgically inaccessible or recurrent tumors and to decrease local recurrence. ${ }^{14}$ The role of postoperative radiation therapy is well defined: literature analyses demonstrated that surgery and adjuvant radiotherapy at a dose of $50 \mathrm{~Gy}$ or more resulted in significantly better local control than surgery alone and radiotherapy should be given even if tumor removal is complete. ${ }^{9,15}$ The metastatic rate of this tumor is $23.4 \%{ }^{16}$ The lungs are the most common metastatic site, followed by the liver, bones, adrenals, and kidneys. ${ }^{16,17}$

Much of the published literature on HPC makes clear that recurrent and metastatic disease is a very common occurrence despite a wide array of initial treatment strategies. ${ }^{3,4,18}$

It is prudent to maintain vigilant radiographic surveillance and staging of all patients with intracranial HPC after treatment, regardless of extent of resection or adjuvant radiation. Chemotherapy does not appear to have an established role in the primary management of these tumors. ${ }^{19}$ However, in patients with disseminated distant metastasis, palliative chemotherapy has been tried.

We have here reported a rare case of HPC that arose in the posterior fossa at two different locations and at different intervals. The unusual location of HPC as in our case often makes it difficult to diagnose via radiologic study alone. Because of relatively high tendency of intraoperative bleeding of HPCs and recurrence even after GTR, the importance of differentiation between HPCs and meningiomas cannot be overemphasized.

\section{Conclusion}

Although extremely rare, a surgeon should be aware of the existence of this disorder to dexterously manage the aggressive nature and high vascular tendency of HPCs.

\section{References}

1 Schiariti M, Goetz P, El-Maghraby H, Tailor J, Kitchen N. Hemangiopericytoma: long-term outcome revisited. Clinical article. J Neurosurg 2011;114(03):747-755
2 Rutkowski MJ, Sughrue ME, Kane AJ, et al. Predictors of mortality following treatment of intracranial hemangiopericytoma. J Neurosurg 2010;113(02):333-339

3 Dufour H, Métellus P, Fuentes S, et al. Meningeal hemangiopericytoma: a retrospective study of 21 patients with special review of postoperative external radiotherapy. Neurosurgery 2001;48(04):756-762, discussion 762-763

4 Guthrie BL, Ebersold MJ, Scheithauer BW, Shaw EG. Meningeal hemangiopericytoma: histopathological features, treatment, and long-term follow-up of 44 cases. Neurosurgery 1989;25(04): 514-522

5 Jääskeläinen J, Servo A, Haltia M, Wahlström T, Valtonen S. Intracranial hemangiopericytoma: radiology, surgery, radiotherapy, and outcome in 21 patients. Surg Neurol 1985;23(03):227-236

6 Stout AP, Murray MR. Hemangiopericytoma: a vascular tumor featuring Zimmermann's pericytes. Ann Surg 1942;116(01):26-33

7 Kim JH, Kwon TH, Kim JH, Park YK, Chung YG, Chung HS. Meningeal hemangiopericytoma: study of 6 cases and review of the literatures. J Korean Neurosurg Soc 2006;39:32-35

8 Alén JF, Lobato RD, Gómez PA, et al. Intracranial hemangiopericytoma: study of 12 cases. Acta Neurochir (Wien) 2001;143(06):575-586

9 Tashjian VS, Khanlou N, Vinters HV, Canalis RF, Becker DP. Hemangiopericytoma of the cerebellopontine angle: a case report and review of the literature. Surg Neurol 2009;72(03): 290-295

10 Rajaram V, Brat DJ, Perry A. Anaplastic meningioma versus meningeal hemangiopericytoma: immunohistochemical and genetic markers. Hum Pathol 2004;35(11):1413-1418

11 Shoji T, Tanaka F, Takata T, et al. Clinical significance of p21 expression in non-small-cell lung cancer. J Clin Oncol 2002; 20(18):3865-3871

12 Barthelmeß S, Geddert H, Boltze C, et al. Solitary fibrous tumors/hemangiopericytomas with different variants of the NAB2STAT6 gene fusion are characterized by specific histomorphology and distinct clinicopathological features. Am J Pathol 2014; 184(04):1209-1218

13 Palkovic S. Multiple recurrence of an intracranial hemangiopericytoma. Neurosurg Rev 1987;10(03):233-236

14 Uemura S, Kuratsu J, Hamada J, et al. Effect of radiation therapy against intracranial hemangiopericytoma. Neurol Med Chir (Tokyo) 1992;32(06):328-332

15 Someya M, Sakata KI, Oouchi A, Nagakura H, Satoh M, Hareyama M. Four cases of meningeal hemangiopericytoma treated with surgery and radiotherapy. Jpn J Clin Oncol 2001;31(11):548-552

16 Mena H, Ribas JL, Pezeshkpour GH, Cowan DN, Parisi JE. Hemangiopericytoma of the central nervous system: a review of 94 cases. Hum Pathol 1991;22(01):84-91

17 Enzinger FM, Smith BH. Hemangiopericytoma. An analysis of 106 cases. Hum Pathol 1976;7(01):61-82

18 Chacko G, Chacko AG, Rajshekhar V, Muliyil JP. Intracranial hemangiopericytomas: correlation of topoisomerase II alpha expression with biologic behavior. Surg Neurol 2006;65(01):11-17

19 Ecker RD, Marsh WR, Pollock BE, et al. Hemangiopericytoma in the central nervous system: treatment, pathological features, and long-term follow up in 38 patients. J Neurosurg 2003; 98(06):1182-1187 